\title{
INDUCED MUSCLE RELAXATION IN THE TREATMENT OF LUMBAR INTERVERTEBRAL DISC LESIONS
}

\author{
ROBERT H. V. HAFNER, F.R.C.S. \\ Consultant Orthopaedic Surgeon to St. Giles' Hospital, \\ London, S.E.5 \\ The Medway and Gravesend Group of Hospitals and \\ the Queen Elizabeth Hospital for Children, London.
}

\author{
Charles D. T. JAmes, F.F.A.R.C.S. \\ Consultant Anaesthetist, St. Giles' Hospital, London, \\ S.E.5 and the King's College Hospital Group.
}

For many years it has been realised that patients suffering from lumbar intervertebral disc lesions who are subjected to a general anaesthetic for some unrelated disorder not infrequently benefit.

A method of treatment was instituted following on this observation and was referred to as "Deep Sleep". We are not absolutely sure who should be credited with the original idea but it was used routinely by Burns and Young from about 1948 onwards.

\section{Method}

The patient was premedicated with 'Omnopon' and soopolamine and deep ether anaesthesia induced for a period of $\frac{1}{2}$ to $\frac{3}{4}$ of an hour. Post operative medication was then given to maintain a drowsy condition for some hours. It was found that this procedure on its own benefited the majority of patients suffering from lumbar intervertebral disc lesions. A small number were unaffected by it and approximately $5 \%$ were made worse. The latter were affected in one of several different ways. Sometimes the pain in the back was made worse and pain in one or ather of the lower limbs exacerbated. Also some patients who before the treatment suffered only from low back pain began now to experience pain down one or other lower limb. Fortunately, this worsening was usually transitory and after a few weeks even these patients were usually better than they had been before the anaesthetic.

After the introduction of muscle relaxants into clinical anaesthesia the original technique was modified, and the procedure now used is as follows:

The patient is premedicated either with Omnopon and scopolamine or morphine and atropine in the usual doses. Anaesthesia is induced with a sleep dose of thiopentone $(2.5 \%)$ and maintained with nitrous oxide and oxygen. Sufficient musole relaxant (usually gallamine) is given intravenously to produce complete muscle paralysis, and respiration is maintained by manual compression of the reservoir bag of the anaesthetic machine. As a result of the generalised muscle paralysis the normal lumbar lordosis flattens spontaneously and the lumbar region of the spine becomes straighter. This can be confirmed by sliding a hand beneath the small of the patient's back.

Fig.1 shows the lumbar region of the spine before relaxant anaesthesia and Fig. 2 the same area after relaxants. It will be seen that the lumbar lordosis has been markedly decreased.
RICHARD ROBERTSHAW, M.B., B.S., D.A. Registrar, Department of Anaesthesia, St. Giles' Hospital, London, S.E.5.

In addition, two other procedures are carried out $\mathbb{C}_{0}$ whilst the patient is anaesthetised. Straight-leg raising 3 is performed on each side followed by flexion of the hips to the abdomen with the knees flexed; Fig. 3 ? shows the complete flattening of the lumbar lordosis $\vec{\infty}$ when this is done. Complete muscle paralysis is maintained for 15-20 minutes before reversal with $\omega$ atropine and prostigmine. It was discovered that this procedure is more successful than the original $\frac{\text { O }}{3}$ "Deep Sleep".

\section{Mechanism}

We look upon "Deop Sleep" as a type of spinal manipulation in which very little force $\vec{\theta}$ is used; perhaps it would be more accuratef described as an auto-manipulation.

We consider that the majority of the patientes we have treated by this method are not suffering from what is usually referred to as a prolapsed intervertebral disc, if one means by this a lesion in which part of the nucleus pulposus has penetrated a ruptured or detached annulus fibrosus and entered the spinal canal. We believe that the majority of these patients are suffering from disc protrusions or herniations of part of the nucleus pulposus into an annulus fibrosus which is stretched but not ruptured or separated from its attachment to the vertebral bodies.

The situation is demonstrated very diagramatically in Figs. 4 and 5 . In the former it will 0 be seen that part of the nucleus pulposus has penetrated the torn annulus fibrosus and now lies in the spinal canal. We consider it unlikely that such a prolapse will revert spontaneously $\sigma$ and although healing may occur over a long $N$ period of time by absorption or fibrosis, this $\hat{\omega}_{\mathrm{N}}$ is the type of case best treated by laminectomy.

Fig. 5 shows part of the pulposus herniating into an annulus fibrosus which is not ruptured or separated from its attachments and thus $\stackrel{\mathscr{C}}{+}$ can still contain the protrusion.

It is this type of case which responds well $\stackrel{\vec{D}}{\vec{D}}$ to conservative measures, such as those des- 


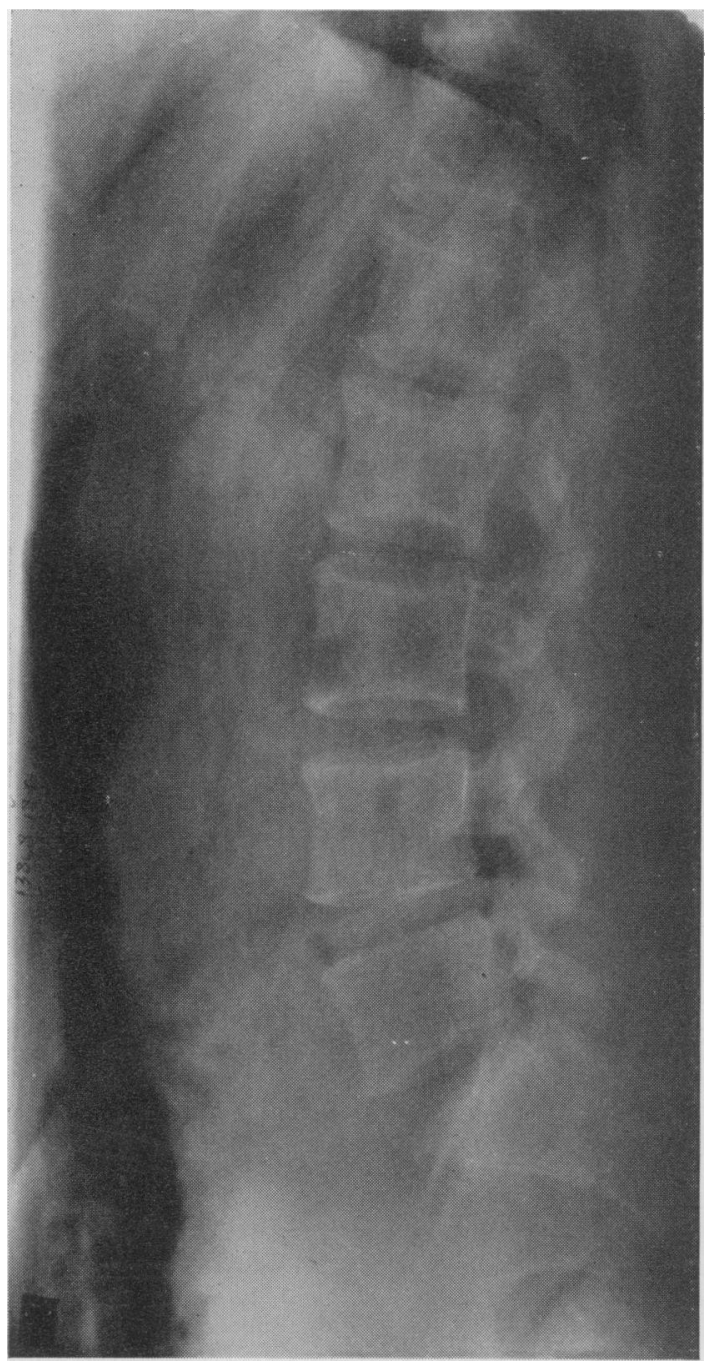

FIG. 1.-Lumbar region of the spine before relaxant anaesthesia.

cribed above, because, given favourable conditions, the herniation can move back from the spinal canal and be held once more by the annulus fibrosus.

The method is not used in isolation but is part of a general system of conservative treatment of intervertebral disc lesions.

The patients thus treated are those with severe or moderately severe lumbar intervertebral disc protrusions which are considered from the onset to be too severe for Out-patient treatment, or who have failed to respond to it. The criteria of severity are the degree of stiffness of the lumbar region of the spine and the amount of limitation of straight leg raising

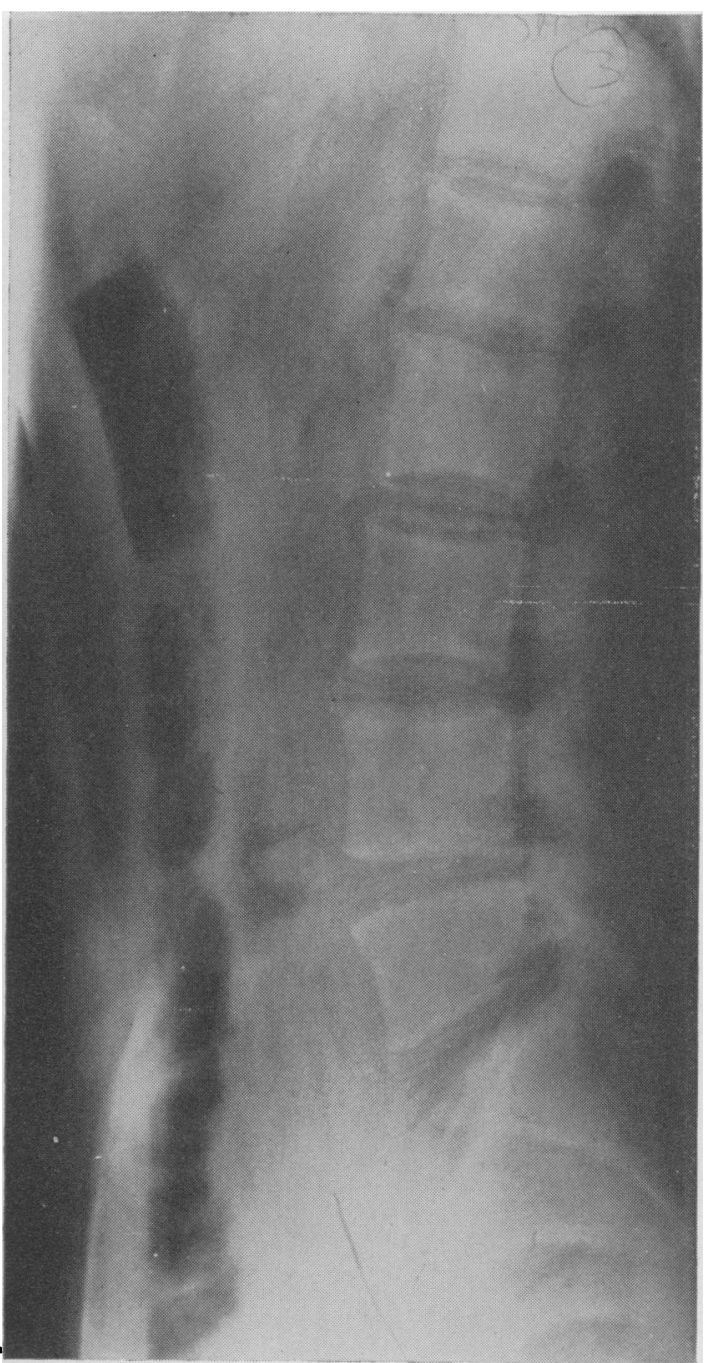

FIG. 2.-Lumbar region after giving relaxants.

together with any abnormal neurological signs which may be present.

$\mathrm{X}$-rays of the lumbar and sacral regions of the spine are taken to exclude bony abnormality or disease and the patient is kept at complete rest in bed with fracture boards beneath the mattress to produce a firm surface. Skin traction is applied to the lower limbs with between 5 and $15 \mathrm{lbs}$. pull on each side, depending on the tolerance of the patient. It is the complete and uninterrupted rest in bed which is the most valuable method of treatment and we do not consider that the traction itself has any very great benerit in the majority of cases but there are two very important reasons for including it. 


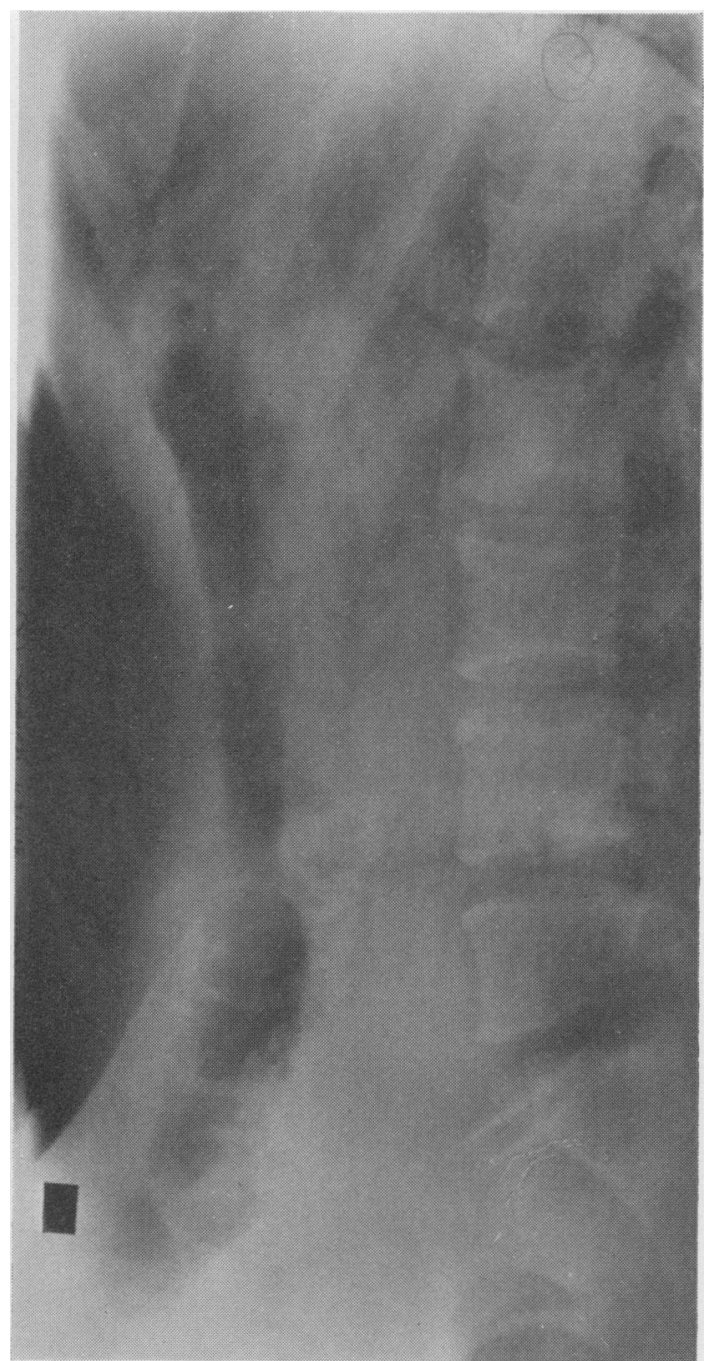

FIG. 3.-Complete flattening of the lumbar lordosis after flexion of hips to abdomen with knees flexed.

Firstly, it keeps the patients strictly confined to bed and, secondly, it is good for morale; they feel that something positive is being done. Analgesics and sedatives are given as required.

At intervals of a few days progress is assessed by the severity of symptoms and by the straight leg raising test. If the patient improves, treatment is continued for two or, if necessary, three weeks after which the traction is removed and the patient turned on to the face 3 to 4 times daily and instructed in hyperextension exercises.

After a few days of this treatment the patient is allowed gradually to sit up and then to start getting out of bed wearing a surgical corset. $\mathrm{He}$ is then discharged to out-patient physiotherapy until fit to return to work.

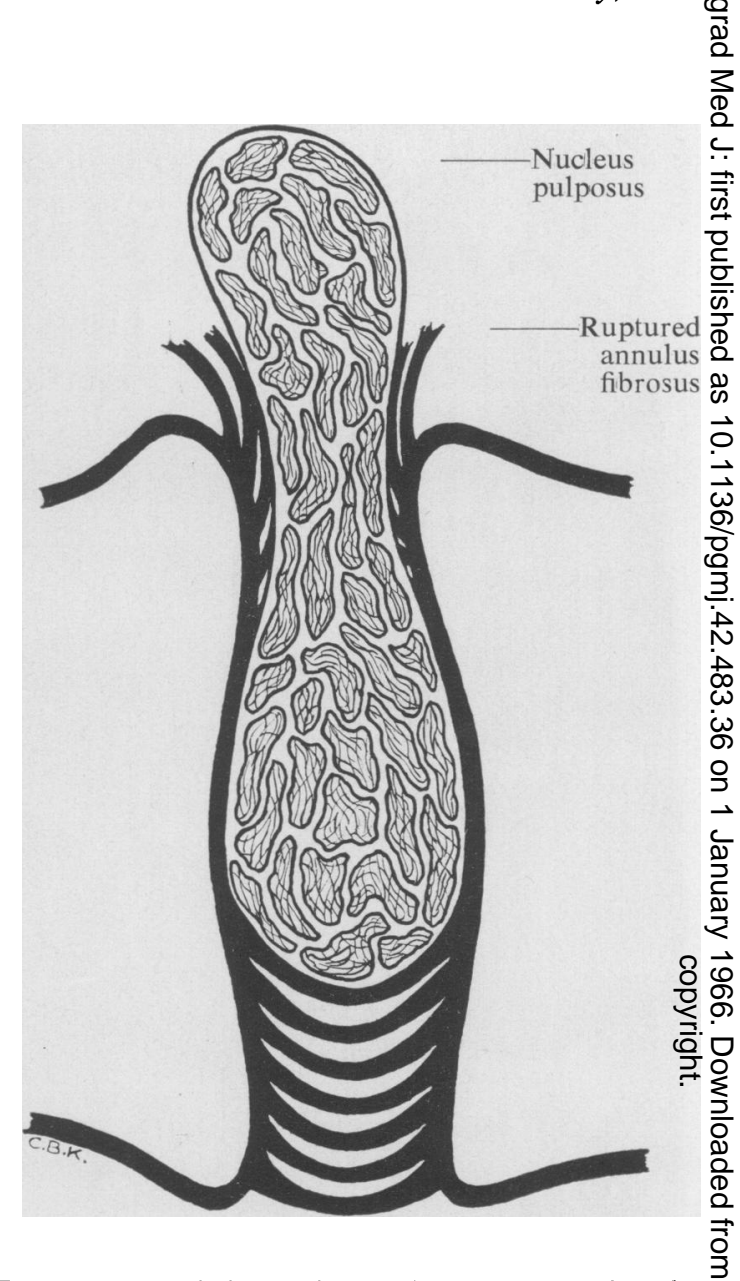

Fig. 4.-Part of the nucleus pulposus penetrating the torn annulus fibrosus and lying in the spinal canal.

The "Deep Sleep" technique as described $\stackrel{3}{3}$. above is used on those patients who fail to improve at all or rapidly enough with the above regime. If necessary it is given on as many as $\stackrel{\circ}{\circ}$ three occasions at weekly intervals. The results are shown in Table 1.

Whilst carrying out this technique one of us $D$ (C.D.T.J.) observed that it was sometimes im- 을 possible, even with the patient completely N relaxed, to achieve full straight-leg raising on the more painful side. We could not understand $\widetilde{N}$ this completely, but we do know that relaxant $\underset{\omega}{N}$ anaesthesia does not completely relieve muscle $\bar{F}$ spasm in patients suffering from trismus com- 6 plicating dental sepsis with which a possible $\underset{\varnothing}{\Phi}$ analogy can be drawn. Musole relaxants act $\stackrel{?}{?}$ on the myoneural junction and not on the muscle fibres themselves.

It is sometimes observed that relaxants alone $\frac{\mathbb{P}}{\mathbb{Q}}$ 


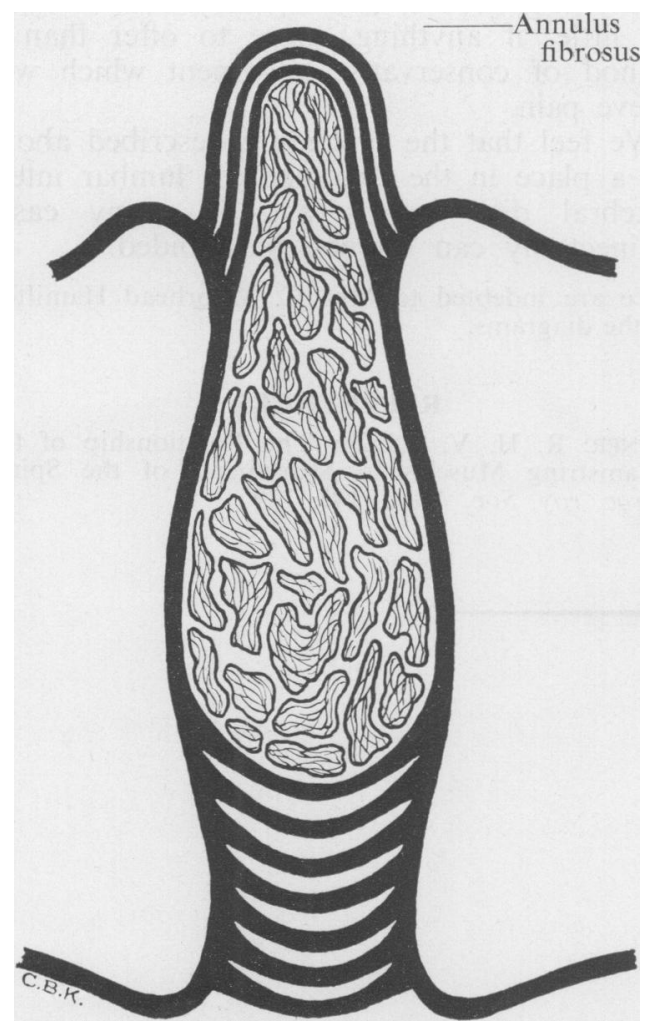

FIG. 5.-Part of the nucleus pulposus herniating into an annulus fibrosus which is not ruptured or separated from its attachments.

do not completely relieve muscle spasm while ether which acts partially on the muscle fibres themselves can produce total flaccidity. Is it that the failure to achieve full straight-leg raising is due to continuing muscle spasm because of nerve root irritation?

We consider that short or tight hamstring muscles are an unlikely cause of this limitation not only because it is unilateral but because previous investigations have shed much doubt on the existence of this syndrome (Hafner, 1952).

\section{Case Reports}

Case No. 1. An example of the dramatic type of response.

Mrs. B. aged 24 years. Teacher of dancing and housewife. She developed sudden severe low back pain in February 1961 when teaching children dancing. With physiotherapy and a surgical corset she recovered in a few months and suffered thereafter only occasional transitory attacks of low back pain until March 1962 when she developed an attack of severe low back pain radiating down her right lower
TABLE 1

$\left.\begin{array}{|c|c|c|c|c|}\hline \begin{array}{c}\text { Total } \\ \text { Ca. of }\end{array} & \text { Male } & \text { Female } & \begin{array}{c}\text { Cases } \\ \text { Manipu- } \\ \text { lated } \\ \text { More } \\ \text { Than } \\ \text { Once }\end{array} & \multicolumn{1}{c|}{\begin{array}{c}\text { Cases } \\ \text { Necessitating } \\ \text { Laminectomy }\end{array}} \\ \hline 126 & \begin{array}{c}\text { Average } \\ \text { age } \\ 37 \text { yrs. }\end{array} & \begin{array}{c}\text { Average } \\ \text { age } \\ 35 \text { yrs. }\end{array} & \begin{array}{l}\text { Male 15 } \\ \text { Female 8 }\end{array} & \begin{array}{l}\text { Male 6 } \\ (4.9 \%) \\ \text { Female 6 } \\ (27 \%)\end{array}\end{array}\right\} 12(15 \%)$

limb to the foot and down her left lower limb as far as the knee. On examination her spine was held rigid and there was a fixed flexion deformity of about $20^{\circ}$ Straight leg raising was right $20^{\circ}$, left $20^{\circ}$.

She was admitted to hospital and given "Deep Sleep" with dramatic improvement. The following day she had no pain and straight leg raising was right $90^{\circ}$, left $90^{\circ}$. A slight recurrence of pain occurred three days later when she leaned back in a wheel chair and the chair back gave way. A second "Deep Sleep" was carried out with equal success and when she was discharged from hospital a few days later she was symptom free.

She had her first baby in December 1962 without inoident and since then has had no further back trouble.

Case No. 2. An example of a patient being made worse by the procedure.

Miss T. aged 25 years. Medical Secretary. In 1954 she began to experience attacks of low back pain following any strenuous activity.

She was treated with a corset and physiotherapy with some benefit but was still unable to engage in rowing or other strenuous physical exertion, neither could she manage without her corset. She sought further treatment in the hope that she would be able to engage in more arduous activities or, at any rate, she would be able to discard her corset. She was therefore admitted to hospital and a "Deep Sleep" procedure carried out 20.1.58. The following day her back pain was much worse and now radiated into both buttocks and lower limbs and was associated with a feeling of pins and needles in both feet. She gradually improved over the course of the next few weeks and she remained largely pain-free until 1962 when she had a recurrence of pain in her back and lower limbs. This responded to a two months course of physiotherapy as an outpatient, since when she has been symptom-free and has discarded her corset but has not resumed rowing.

\section{Summary}

This paper concerns 126 patients of whom 81 were males with an average age of 37 years, and 45 females with an average age of 35 years. All were suffering from lumbar intervertebral disc lesions sufficiently severe to warrant inpatient treatment with the exception of Miss $T$. the second of the two illustrative cases, who was on the staff of one of the hospitals and would not in the ordinary way have been considered severe enough to warrant in-patient therapy. 
Of these 126 patients $4.9 \%$ of males required a laminectomy, and no less than $27 \%$ of females, giving a total of $15 \%$. We feel that a major factor in this surprising disparity may well be that there were so many fewer females than males in the series.

We do not wish to decry the value of laminectomy in the treatment of severe lumbar intervertebral disc lesions and would advise surgery in such cases if they failed to respond appreciably to a week or two of conservative in-patient therapy. Unfortunately, one of the limitations of laminectomy is that one cannot give the patient a guarantee of being able to resume heavy work thereafiter and if a change over to light work is obligatory then surgery has little, if anything, more to offer than a method of conservative treatment which will relieve pain.

We feel that the procedure described above has a place in the treatment of lumbar intervertebral disc lesions and in many cases laminectomy can thereby be avoided.

We are indeblted to Mrs. C. Moorhead Hamilton for the diagrams.

\section{REFERENCES}

HAFNER, R. H. V. (1952): The Relationship of the Hamstring Muscles to Movements of the Spine, Proc. roy. Soc. Med., 145, 139. 